\title{
Chronologie du développement et évolution du stockage de calcium et des cellules à urates chez Niphargus schellenbergi Karaman. *
}

\author{
par
}

\author{
François GRAF et Philippe MICHAUT **
}

\author{
SUMMARY
}

\section{Developmental chronology and evolution of calcium storage and urate containing cells in Niphargus schellenbergi Karaman.}

The intra-marsupial development of Niphargus schellenbergi is divided into three phases: within the chorion, embryonic intermoult and juvenile intermoult. The disappearance of the chorion divides the first phase from the second. A double exuviation exists between the last two. The chronology of the embryonic development and of the beginning of post-embryonic development is established. On hatching the juvenile N.schellenbergi has one pair of hepatopancreatic caeca. During the first post-embryonic intermoults are formed the ventral hepatopancreatic caeca, posterior caeca and anterior caecum. The relation between posterior caeca and the aorta is described.

The study of calcium storage before moulting showed that calcareous concretions located in posterior caeca and midgut have the typical form, volume, quantity and distribution of the species. This calcium accumulation process occurs in the midgut following the first postembryonic exuviation. It is only when the sixth exuviation is attained that storage in the posterior caeca is similar to the one in adults.

The urate containing cells located at the lower face of the pericardial septum begin to accumulate urate spherules 15 days before hatching. They vary in form, volume, localization and composition, and so may be considered as storage sites for urate, pigments and various ions (P, $\mathrm{K}, \mathrm{Ca}, \mathrm{S}, \mathrm{Cl}, \mathrm{Na}, \mathrm{Mg}$ ) which are returned to circulation.

Il est désormais bien établi qu'avant la mue le Crustacé hypogé Niphargus stocke dans son intestin moyen et dans ses caecums postérieurs des concrétions calcaires qui seront utilisées après l'exuviation pour la consolidation de la nouvelle cuticule. Ce processus de stockage de calcium avant la mue, qui s'observe également chez le Talitridé aérien Orchestia (Graf, 1962, 1969) et le Mysidacé Spelaeomysis longipes (Nath, 1972), est en rapport avec le mode de vie du Crustacé. Chez Orchestia ce phénomène de stockage de calcium apparait au cours de la vie embryonnaire (Graf, 1972); la présente étude a pour but de rechercher à quel stade embryonnaire ou

* Une partie de cette étude a fait l'objet d'une communication lors du 3ème colloque international sur les genres Gammarus et Niphargus et du 2ème symposium international sur l'écologie des eaux souterraines (Schlitz, 22 - 26 sept. 1975).

** Laboratoire de Biologie animale et générale, Faculté des Sciences de la Vie et de l'Environnement, 6, Boulevard Gabriel, 21000 - Dijon, France. 
post-embryonnaire ce stockage intervient chez Niphargus, ce qui nécessite d'etablir la chronologie du développement de cet Amphipode hypogé. Par ailleurs, ayant montré la présence de cellules à urates chez ce Crustacé (Graf, 1971), il apparaissait particulièrement intéressant de conduire cette étude sur des individus d'une population de Niphargus schellenbergi chez lesquels ces cellules sont particulièrement nombreuses.

Après avoir établi les grandes étapes du développement de ce Niphargus, nous envisagerons d'une part le développement des dérivés de l'intestin moyen et le stockage de calcium et d'autre part l'apparition et l'evolution des cellules à urates.

\section{LES GRANDES ETAPES DU DEVELOPPEMENT. \\ Si l' Travaux antérieurs sur Niphargus virei}

Si l'embryologie des Amphipodes épigés a fait l'objet d'assez nombreux travaux (cf. biblio. in Weygolt, 1958; Ginet, 1960, Bregazzi, 1973), les données concernant le développement de Niphargus sont relativement restreintes. Husson (1949) fait état du ralentissement du développement des Amphipodes hypogés comparativement à celui des épigés et précise (1959) que, chez $N$. virei, il faut compter environ 4 mois de vie marsupiale (de la ponte à la mise-bas) alors que chez une plus petite espèce (N.aquilex Schiödte) il faut une cinquantaine de jours pour obtenir l'eclosion. Chez N.virei la première mue se fait en moyenne un mois environ après la mise-bas, les mues suivantes n'ayant lieu que tous les 2 ou 3 mois (Husson, 1953). Selon Ginet (1960), la gestation de $N$.virei se subdivise en 2 phases séparées par l'éclosion: l'incubation dure de 70 à 90 jours et la vie marsupiale jusqu'à 40 jours, ce qui fait un total de 4 mois. Les intermues sont en moyenne de 70 jours pendant la première année et de 110 jours pendant la seconde.

Les travaux de Ginet (1960) et Turquin $(1967,1969)$ précisent la chronologie de la morphogenèse de $N$.virei, la période d'incubation étant scindée en 4 phases d'une vingtaine de jours:

- 0 à 25 jours: de la ponte à l'image nauplienne (segmentation, édification de la bande germinative et de l'organe dorsal).

- 25 à 50 jours: du stade nauplien à la fin de la mise en place des appendices. Cette période voit notamment la mue nauplienne, le stade métanauplien, la courbure ventrale, l'apparition d'une coque albumineuse.

- 50 à 70 jours: segmentation des appendices et du corps. Vers 60 jours, formation de l'intestin provisoire, développement maximal de l'organe dorsal, l'embryon mue une nouvelle fois (Turquin, 1967). L'embryon est achevé à 70 jours.

- 70 à 90 jours: différenciation définitive, acquisition des mouvements du coeur puis du corps. L'organe dorsal régresse dès 80 jours. L'éclosion s'effectue par rupture du chorion.

$\mathrm{Si}$ ces diverses étapes se retrouvent pour l'essentiel chez N.schellenbergi, nos observations divergent cependant, d'une part sur la présence d'une nouvelle mue entre la mue nauplienne et l'éclosion, et, d'autre part, sur la persistance du chorion jusqu'à l'éclosion. 


\section{B - Observations sur Niphargus schellenbergi.}

Cette étude est menée sur des $N$. schellenbergi [espèce considérée par Straskraba (1972) comme indépendante de N.aquilex Schiödte] récoltés dans une source très intermittente de la commune d'Avot (Côte-d'Or) en contre-bas d'un verger cadastré A 328.

Les oeufs de $N$. schellenbergi, espèce de taille moyenne, sont semblables à ceux de $N$.virei décrits par Ginet (1960) quant à leur coloration jaune pâle et à leurs dimensions $(0,7 \mathrm{~mm} \times 0,6 \mathrm{~mm}$ en début d'incubation; $0,8 \times 0,65$ avant l'éclosion). Comme chez les autres Gammaridés, le nombre des oeufs est fonction de la taille de la femelle, il est en moyenne d'une douzaine pour les femelles dont la taille voisine $9 \mathrm{~mm}$, ce qui est légèrement supérieur au cas de femelles de $N$. longicaudatus de 10 à $11 \mathrm{~mm}$ chez lesquelles il y a en moyenne 9 oeufs (Ginet, 1960).

L'observation du développement est effectuée, d'une part sur des oeufs prélevés périodiquement dans le marsupium de plusieurs femelles (récoltées gestantes ou ayant pondu en élevage) et, d'autre part, sur des oeufs placés en incubation artificielle dans des salières dont l'eau est renouveláe tous les 2 jours; si cette dernière méthode est particulièrement satisfaisante, il est cependant délicat d'éviter diverses infections de l'oeuf au cours d'un développement complet, ce qui oblige à considérer que le rôle du marsupium ne doit pas être limité à celui d'un simple support. Notons le comportement original d'une femelle qui a très rapidement réintroduit dans son marsupium un oeuf qui venait d'en être extirpé à l'aide d'un pinceau fin.

La vie marsupiale de $N$. schellenbergi, d'une durée moyenne de 100 jours (élevage à $10-12^{\circ} \mathrm{C}$ ), peut, comme pour le Talitridé Orchestia cavimana, être scindée
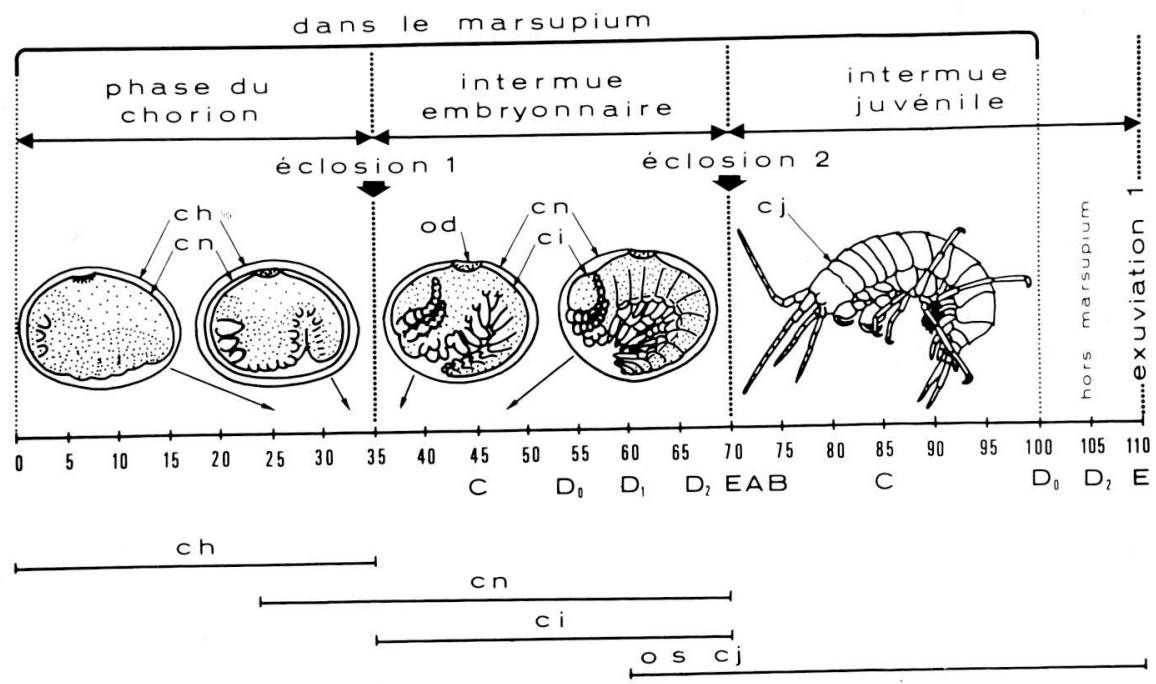

Fig. 1. Chronologie, en jours, du développement de $N$. schellenbergi fondée sur l'évolution des enveloppes embryonnaires et des formations cuticulaires. Explications dans le texte. $c h$, chorion; $c n$, cuticule nauplienne; $c i$, cuticule intermédiaire; $\mathrm{o}, \mathrm{s}, \mathrm{cj}$, ongles, soies, cuticule juvéniles; od, organe dorsal. 
en 3 étapes (Graf, 1972). Les deux premières étapes, nommées phase du chorion et intermue embryonnaire, correspondent au développement embryonnaire proprement dit (35 jours chacune) et sont séparées par la rupture et la disparition du chorion. La troisième étape, d'environ 30 jours, correspond à la plus grande partie de la première intermue post-embryonnaire (nommée intermue juvénile), les pulli étant libres dans le marsupium (fig. 1)

1. Première étape: phase du chorion ( 35 jours).

- 0 à $12 \mathrm{j} .:$ phase de segmentation de l'oeuf à l'intérieur du chorion.

- $13 \mathrm{j}$.: édification de la bande germinative.

- $16 \mathrm{j}$.: édification de l'organe dorsal qui n'adhère pas au chorion.

- $20 \mathrm{j}$.: stade nauplien qui sera rapidement entouré d'une mince enveloppe, la cuticule nauplienne.

- 25 j.: stade métanauplien caractérisé par l'allongement et l'ébauche de la métamérisation du futur mésosome.

- $27 \mathrm{j}$.: début de la courbure ventrale entre mésosome et pléon; antennes et mandibules sont nettement visibles.

- $32 \mathrm{j}$.: alors que la courbure ventrale est maximale et que les ébauches des appendices du mésosome apparaissent, la cuticule nauplienne se décolle de l'embryon sauf au niveau de l'organe dorsal (fig. 1).

- $35 \mathrm{j}$.: le chorion se rompt et disparait. Cet instant peut être considéré comme une éclosion primaire car l'embryon n'est alors enfermé que dans une enveloppe, la cuticule nauplienne, qu'il a lui-même sécrétée. Sous la cuticule nauplienne l'ectoderme embryonnaire élabore une nouvelle enveloppe nommée cuticule intermédiaire (Graf, 1972). La rupture du chorion est attestée, d'une part par la constatation du rejet de cette membrane sur des oeufs placés en incubation artificielle et, d'autre part, du fait que, dès cet instant l'organe dorsal débouche à l'extérieur et qu'il est alors possible de voir des extrusions par le centre de cet organe, extrusions peut-être dues aux conditions d'observation, à l'influence de la chaleur ou de la lumière. Observé en premier chez le Talitridé Orchestia (Graf, 1972) et confirmé chez des Lysianassidés (Bregazzi, 1973), ce processus d'éclatement du chorion constaté chez le Gammaridé hypogé $N$. schellenbergi pourrait être général aux Amphipodes, à moins qu'il ne soit en relation avec le mode de vie assez particulier des espèces considérées: mode de vie aérien pour Orchestia, 6 mois d'incubation à - $1^{\circ} \mathrm{C}$ pour les Lysianassidés des eaux de l'Antarctique, longue incubation aussi pour Niphargus.

\section{Deuxième étape: intermue embryonnaire (35 jours).}

Cette phase d'organogenèse se déroule entre deux exuviations particulières (d'où l'emploi du terme d'intermue), d'une part après le décollement de la cuticule nauplienne et la disparition du chorion (éclosion 1) et, d'autre part, avant le rejet des cuticules nauplienne et intermédiaire correspondant à la seconde éclosion, à la suite de laquelle le jeune sera libre dans le marsupium (fig. 1).

-37 j.: les antennes sont segmentées, les ébauches de tous les appendices sont présentes. 
- $45 \mathrm{j} .:$ le corps et les appendices sont segmentés, l'intestin moyen provisoire délimite entièrement le vitellus (planche $\mathrm{I}, 1$ ).

- $50 \mathrm{j}$.: édification du coeur et d'une paire de caecums hépatopancréatiques qui s'isolent de l'intestin provisoire par étranglement de ses parois latéro-ventrales. Les branchies et les plaques coxales s'individualisent.

- $55 \mathrm{j}$.: la cuticule intermédiaire se décolle de l'épiderme (stade D O de l'intermue embryonnaire). Début d'accumulation d'urates dans les futures cellules à urates.

$-58 \mathrm{j}$. : contractions cardiaques irrégulières et intermittentes. Contractions péristaltiques des caecums hépatopancréatiques et de l'intestin moyen sous l'action de la lumière d'observation.

$-59 \mathrm{j} .:$ contractions cardiaques régulières mais intermittentes.

- 60 j.: différenciation du matériel fibrillaire des matrices des soies des ongles (stade D 1' de l'intermue embryonnaire). Les matrices trichogènes sont reliées à la cuticule intermédiaire par une fibre nerveuse, comme pour Orchestia (Graf, 1972). Début d'édification des caecums postérieurs par évagination de la région dorsale de l'extrémité postérieure de l'intestin moyen (planche I, 2).

- 62 j.: la sécrétion des étuis des ongles et des soies commence (stade D 1"). Contractions musculaires dans la région antérieure du corps.

$-63 \mathrm{j}$.: contractions cardiaques régulières et constantes. Mouvements du corps. Début de régression de l'organe dorsal.

- $66 \mathrm{j}$.: début de sécrétion de la couche préexuviale du futur squelette, la cuticule juvénile (stade D 2 de l'intermue embryonnaire; planche I, 3).

$-68 \mathrm{j} .:$ mouvements du corps assez vifs.

- 70 j.: éclosion secondaire et libération du jeune dans le marsupium par rupture et rejet des cuticules nauplienne et intermédiaire. A l'éclosion 2, le jeune $N$. schellenbergi mesure environ 1,9 $\mathrm{mm}$ (de la base des antennes au telson), le flagelle principal des antennes 1 comporte 6 articles, celui des antennules (A 2) 4 articles. Il n'est pourvu que d'une seule paire de caecums hépatopancréatiques (dorsaux) qui renferment des réserves vitellines, comme la région médiane de l'intestin moyen (planche I, 4). Les caecums postérieurs sont au tout début de leur développement.

3. Troisième étape: première intermue post-embryonnaire ou intermue juvénile.

Après l'éclosion 2, le jeune N. schellenbergi demeure environ 30 jours dans le marsupium. Il en sortira lorsqu'il sera à l'étape $\mathrm{D} 0$ de cette intermue post-embryonnaire qui se terminera 10 jours après par la première exuviation extra-marsupiale. En fait la sortie du marsupium des jeunes d'une même portée peut être très échelonnée et varier de 18 à 35 jours après l'éclosion 2, mais, dans ces divers cas, la durée de la première intermue post-embryonnaire sera cependant d'environ 40 jours. Les pulli qui sortent du marsupium, alors qu'ils sont parvenus à un stade d'intermue entre C et D 1", s'alimentent dès leur sortie; ceux qui ne sortent qu'en D 1" ne s'alimentent pas avant la première exuviation extra-marsupiale, les concrétions calcaires apparaissant dès ce stade d'intermue dans leur intestin moyen. Les réserves de vitellus de l'intestin moyen sont utilisées dans les jours qui suivent l'éclosion, alors que celles contenues dans les caecums hépatopancréatiques peuvent, dans certains cas, persister partiellement jusque durant l'intermue 2. Cette troisième phase du développement voit l'organogenèse se poursuivre. 


\section{Etapes suivantes.}

Après leur sortie du marsupium, les jeunes sont élevés, soit en présence d'argile (dans laquelle ils creusent très rapidement des terriers), soit en présence de feuilles mortes d'Orme et reçoivent dans ces deux cas une alimentation carnée. Contrairement à ce qui a été observé chez N.virei par Ginet (1955) et Gounot (1960), les individus élevés en l'absence de limon argileux se développent aussi bien que ceux qui disposent de ce sédiment: l'argile ne conditionne donc pas la survie ou le développement des individus de cette population de $N$. schellenbergi.

Durant la première année, les intermues sont en moyenne de 70 à 90 jours (fig. 2). Les durées relatives des diverses étapes et stades d'intermue sont semblables à celles de l'adulte et identiques à celles de N.virei (Graf, 1968), à savoir que la période $\mathrm{D}$ correspond à environ $20 \%$ de l'intermue totale.

L'accroissement de taille est relativement faible au cours des premières intermues: 1,9 mm à l'intermue 1,2,4 mm à l'int.2, 2,8 mm à l'int. 3, 3,25 mm à l'int. $4,3,7 \mathrm{~mm}$ à l'int. 5 , les plus grands mâles récoltés mesurant $14 \mathrm{~mm}$, les plus grandes femelles $11 \mathrm{~mm}$.

Afin de rechercher un critère permettant de définir à quelle intermue est parvenu un individu, il convenait de savoir comment s'effectue l'augmentation du nombre des articles du flagelle principal des antennes et de celui des antennules. Chez $N$.virei, le flagelle principal des antennes possède 6 articles à l'éclosion, 10 à la mue suivante et en acquiert régulièrement 2 à chaque mue, mais à partir de 18 articles la régularité est perturbée (Ginet, 1960). Chez les jeunes $N$. schellenbergi, le nombre des articles étant aussi souvent impair que pair, nous avons suivi la croissance des flagelles antennaires pendant 5 intermues sur une quarantaine d'individus provenant de 6 portées. Ces jeunes sont élevés par portée et sont observés périodiquement au microscope pour déterminer leur stade d'intermue. A l'approche de la mue, les individus sont isolés; l'observation des flagelles antennaires permet de reconnaitre l'article qui se divise, ce qui est vérifié, après l'exuviation, par la comparaison de l'exuvie et de la nouvelle antenne. Chez $N$. schellenbergi, le nombre d'articles des

\section{PLANCHE I.}

1 - Embryon de $N$. schellenbergi d'environ 45 jours, soit 10 jours après l'éclatement du chorion. L'enveloppe la plus externe correspond à la cuticule nauplienne $(\mathrm{cn})$, le centre de l'organe dorsal $(o d)$ est en communication directe avec le milieu extérieur, l'épiderme est recouvert par la cuticule intermédiaire. L'intestin provisoire ( $\mathrm{imp}$ ) renferme la totalité du vitellus. Les appendices sont segmentés.

2 - Embryon de 60 jours, soit 10 jours avant l'éclosion 2. Le vitellus est essentiellement localisé dans les caecums hépatopancréatiques dorsaux $(c h d)$.

3 - Extrémité d'un péréiopode d'un embryon un peu plus âgé que celui de la figure précédente, soit 4 à 5 jours avant l'éclosion 2 . La formation de l'ongle et le début de sécrétion de la cuticule juvénile $(c j)$ sous la cuticule intermédiaire $(c i)$ permettent de préciser que l'embry on est parvenu au début de l'étape D 2 de l'intermue embryonnaire.

4 et 5 - Coupes tranversales de jeunes $N$. schellenbergi aussitôt après l'éclosion 2 . Le septum péricardique $(s)$ apparait constitué de 2 lames qui doublent la tunique musculaire $(\mathrm{tm})$ du coeur $(c o)$. On note la présence de vitellus $(v)$ dans l'intestin moyen $(\mathrm{im})$ et dans les caecums hépatopancréatiques dorsaux $(c h d) ; c j$, cuticule juvénile; $c m$, connexion mésodermique; $g$, ébauche de gonade; $l s d$, lame septale dorsale; $l s v$, lame septale ventrale; $m i$, mésoderme intestinal. 

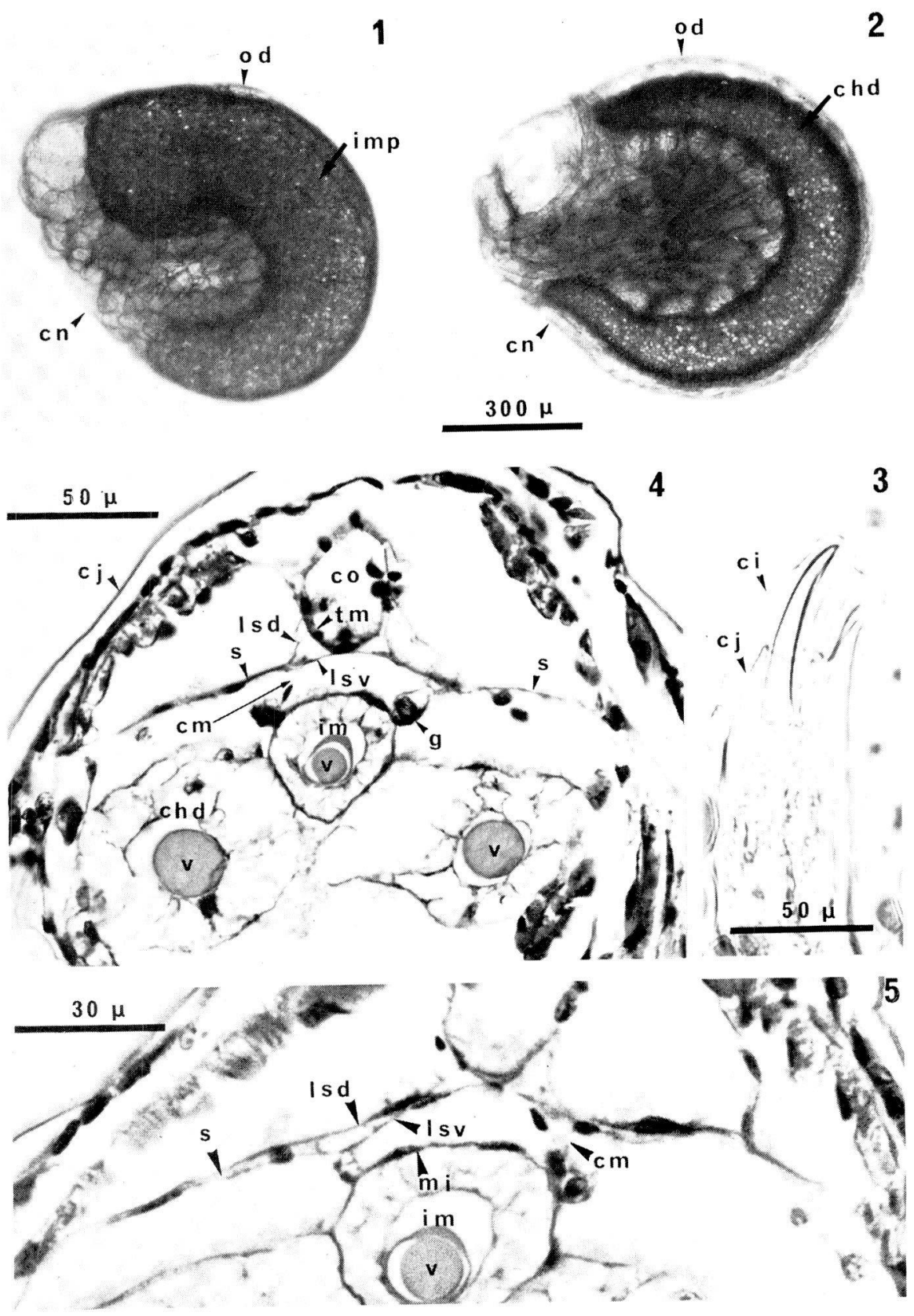


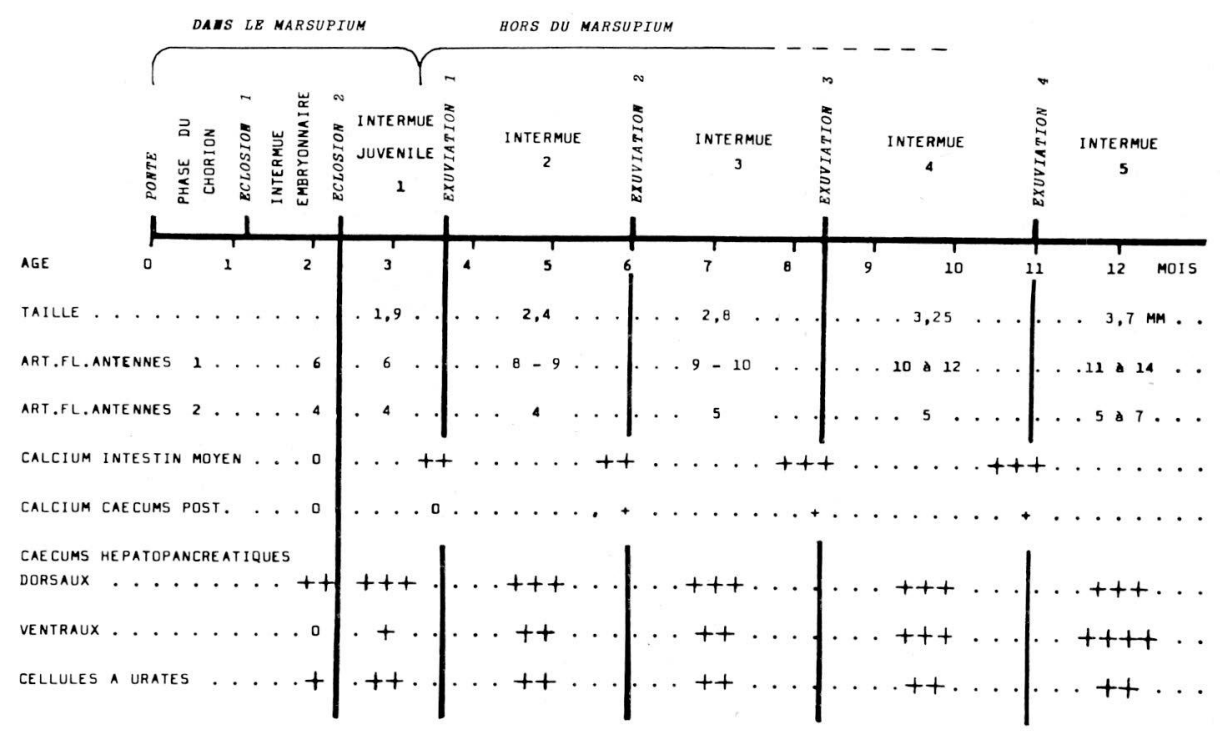

Fig. 2. Tableau récapitulatif de l'évolution de la taille, du nombre des articles des flagelles des antennes 1 et 2, du stockage de calcium, des caecums hépatopancréatiques et des cellules à urates chez $N$. schellenbergi au cours des 6 premières intermues.

flagelles antennaires est, à l'éclosion, de 6 pour les $\mathrm{A} 1$ et de 4 pour les A 2; il est de 25 et 11 chez les plus grands mâles observés $(14 \mathrm{~mm})$ et de 23 et 10 chez les femelles $(11 \mathrm{~mm})$. En ce qui concerne les A 1, la numérotation des articles est faite de l'extrémité proximale (base) vers l'extrémité distale. Lors de l'exuviation 1 (entre les intermues 1 et 2) le nombre des articles passe de 6 à 8 chez les $3 / 4$ des individus par subdivision des articles 3 et 4, et de 6 à 9 chez les autres par subdivision des articles 2, 3 et 4 . Lors des exuviations suivants, seuls les articles proximaux (basaux) 1 et (ou) 2 se subdiviseront. La figure 3 montre les diverses modalités de l'accroissement du nombre de ces articles: ou bien aucun article ne se divise, ou bien la division affecte un seul article (le 1 ou le 2) ou 2 articles (le 1 et le 2). Lors de l'intermue 5, les individus sont répartis en 4 lots subégaux à $11,12,13$ ou 14 articles. Ces irrégularités dans l'accroissement du nombre des articles s'observent non seulement à l'intérieur d'une même portée mais encore sur un même individu, chacun des deux flagelles passant par exemple de 10 à 11 par subdivision de l'article 1 d'un flagelle et subdivision de l'article 2 de l'autre flagelle. Il est de même fréquent que les deux flagelles n'aient pas le même nombre d'articles, ceci indépendamment d'une éventuelle amputation. En ce qui concerne le flagelle des A 2, il présente 4 articles à l'éclosion et durant les intermues 1 et 2, 5 articles durant les intermues 3 et 4 (l'article proximal s'étant divisé); au cours des intermues suivants, l'accroissement devient irrégulier du fait de la division de 0 , de 1 ou de 2 articles. Afin de déterminer à quelle intermue est parvenu un jeune $N$. schellenbergi il conviendra 
donc de considérer à la fois la taille de l'individu et le nombre des articles flagellaires des A 1 et des A 2 (fig. 2).

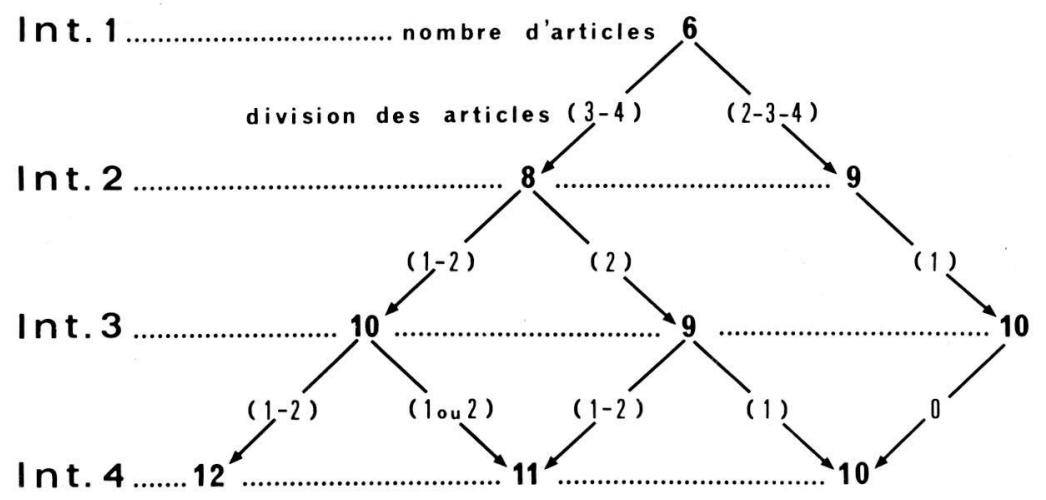

Fig. 3. Diverses modalités de l'accroissement du nombre des articles du flagelle principal des antennes $1 \mathrm{de} N$. schellenbergi au cours des trois premières intermues. La numérotation des articles est faite de l'extrémité proximale (base) vers l'extrémité distale.

\section{EVOLUTION DE L'INTESTIN MOYEN ET DU STOCKAGE DE CALCIUM.}

Nous allons d'abord préciser les grandes étapes de l'organogenèse des dérivés de l'intestin moyen, puis nous envisagerons quelle est la relation anatomique des caecums postérieurs avec l'aorte et comment évolue le processus de stockage de calcium avant la mue.

\section{A - Les dérivés de l'intestin moyen}

Chez les Gammaridés, trois formations glandulaires sont en relation directe avec l'intestin moyen dont elles dérivent:

- deux paires de caecums hépatopancréatiques débouchant ventralement à la limite antérieure de l'intestin moyen. Une paire est ventrale par rapport au tube digestif, elle s'étend jusque dans l'urosome; l'autre paire dite dorsale, en fait latérale par rapport au tube digestif, est légèrement plus courte que la précédente (planche II, 2).

-un caecum antérieur impair débouchant dorsalement à la limite antérieure de l'intestin moyen.

-une paire de caecums postérieurs débouchant dorsalement à la limite postérieure de l'intestin moyen et s'étendant dans le métasome.

Chez $N$. schellenbergi, l'organogenèse du tube digestif et de ses dérivés débute au cours de l'intermue embryonnaire et se poursuit longtemps après l'éclosion. Vers 45 jours, l'endoderme enveloppe le vitellus pour constituer un intestin moyen provisoire (planche I, 1) qui sera à l'origine de l'intestin moyen définitif, des caecums 
hépatopancréatiques, des caecums postérieurs et du caecum antérieur. Vers 50 jours, le tube endodermique se subdivise longitudinalement en trois compartiments, un compartiment médio-dorsal correspondant à l'intestin moyen définitif et deux compartiments latéro-ventraux plus volumineux qui contiennent la plus grande partie du vitellus et correspondent aux caecums hépatopancréatiques (future paire dorsale) (planche I, 2). L'édification des caecums postérieurs débute vers 60 jours selon deux évaginations dorsales de l'extrémité postérieure de l'intestin. Quant au caecum antérieur, il commence à s'organiser, juste avant l'éclosion, à partir d'une évagination médio-dorsale de l'extrémité antérieure de l'intestin. Ainsi, lors de l'éclosion 2, le jeune $N$. schellenbergi n'est pourvu que d'une seule paire de caecums hépatopancréatiques et les caecums antérieur et postérieurs sont à peine ébauchés. La présence d'une seule paire de caecums hépatopancréatiques à l'éclosion a déjà été signalée chez N.virei par Turquin (1967, cet auteur parlant en fait d'une paire de caecums antérieurs) et chez Orchestia par Graf (1972), alors que chez Gammarus les deux paires sont présentes à la naissance.

L'évolution des caecums, relativement lente, est schématisée sur la figure 4. Les caecums hépatopancréatiques ventraux commencent à s'organiser, au cours de l'intermue juvénile 1, à partir de la région antéro-interne des caecums dorsaux; ils s'allongent progressivement vers l'arrière et deviendront plus longs que les caecums dorsaux lors de l'intermue 5 (12 mois environ). Le vitellus contenu dans l'intestin moyen disparait au début de l'intermue juvénile 1, celui contenu dans les caecums hépatopancréatiques sera résorbé beaucoup plus lentement tout au long de cette intermue (planche I, 4). L'allongement des caecums postérieurs vers l'avant s'effectue pour l'essentiel en 4 mois (exuviation 2), mais ne sera maximal qu'au cours de l'intermue 5.

\section{PLANCHE II.}

1 - Coupe transversale au niveau du coeur d'un $N$. schellenbergi adulte montrant la localisation des cellules à urates $(c u)$ sous le septum péricardique $(s)$ subdivisé en 2 lames au niveau du coeur $(\mathrm{co})$; $\mathrm{im}$, intestin moyen; $l s d$, lame septale dorsale; $l s v$, lame septale ventrale; $t m$, tunique musculaire cardiaque.

2 - Coupe transversale au niveau du 7ème mésosomite d'un $N$. schellenbergi adulte montrant la localisation des cellules à urates $(\mathrm{cu})$ au milieu de cellules adipeuses sous le septum péricardique $(s)$; $C c h d$, caecum hépatopancréatique dorsal; $c h v$, caecum hépatopancréatique ventral; $i m$, intestin moyen; $v s$, vésicules séminales. Les caecums postérieurs $(c p)$ ne sont pas localisés dans la lumière de l'aorte postérieure $(a p)$ mais, comme le montre la photographie

3 (agrandissement d'un caecum de la photo 2), entre la lame septale dorsale (lsd) et la paroi de l'aorte.

4 - Coupes sériées réalisées dans la région distale des caecums postérieurs montrant que les caecums sont localisés entre la lame septale dorsale $(l s d)$ et la paroi de l'aorte $(p a)$ et non dans la lumière de l'aorte $(a p)$.

5 - De même chez $N$. virei les caecums postérieurs ( $c p$ ) qui semblent être logés dans la lumière de l'aorte postérieure $(a p)$ sont en fait localisés entre la lame septale dorsale $(l s d)$ et la paroi de l'aorte $(p a) ; \mathrm{cm}$, connexion mésodermique; $i m$, intestin moyen; lsv, lame septale ventrale; $s$, septum péricardique.

6 - Chez $N$. foreli les caecums postérieurs ( $c p$ ) renferment lors de l'exuviation de volumineux sphérolithes et les cellules à urates sont le plus souvent, au niveau du métasome. alignées le long des caecums. 


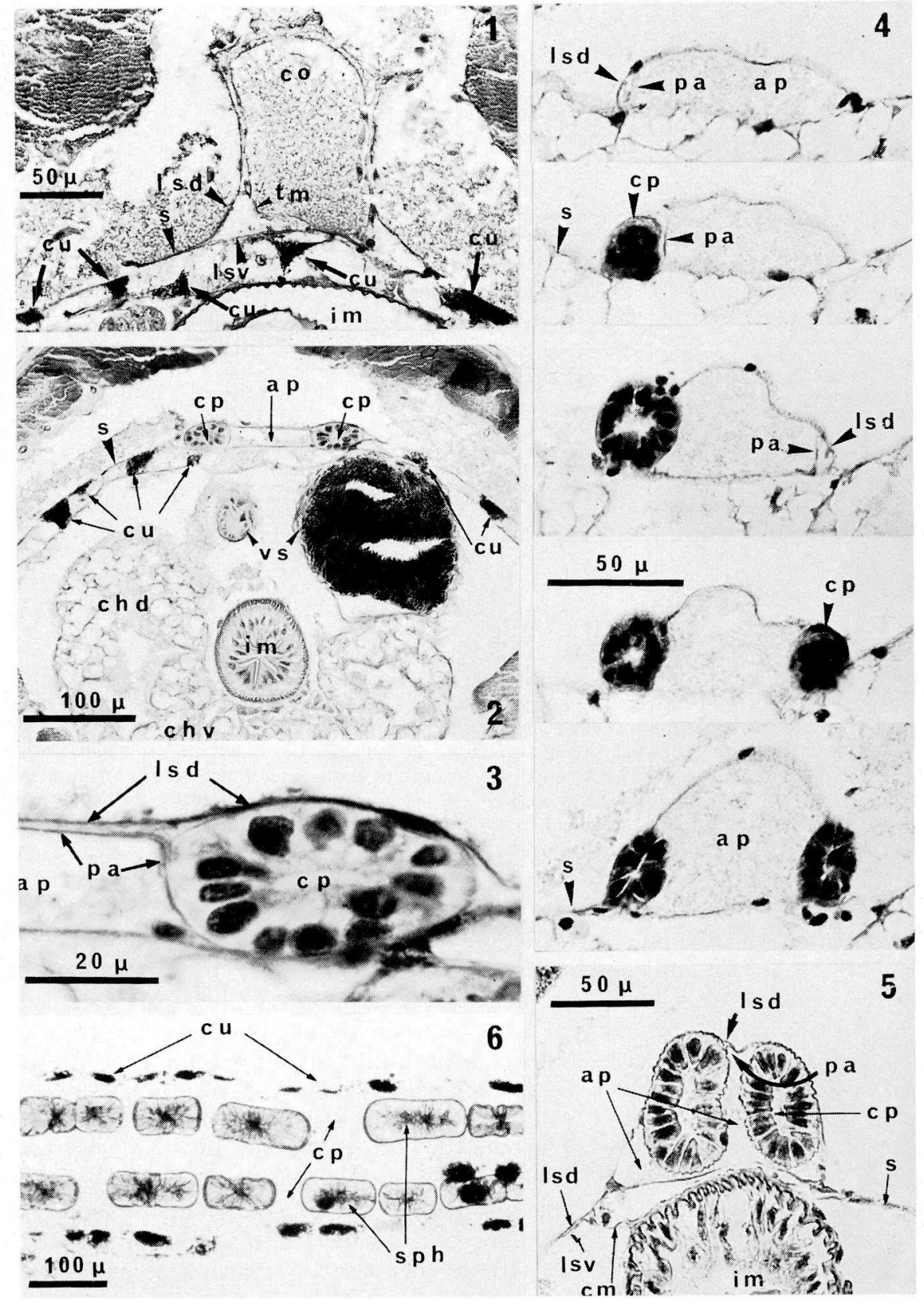



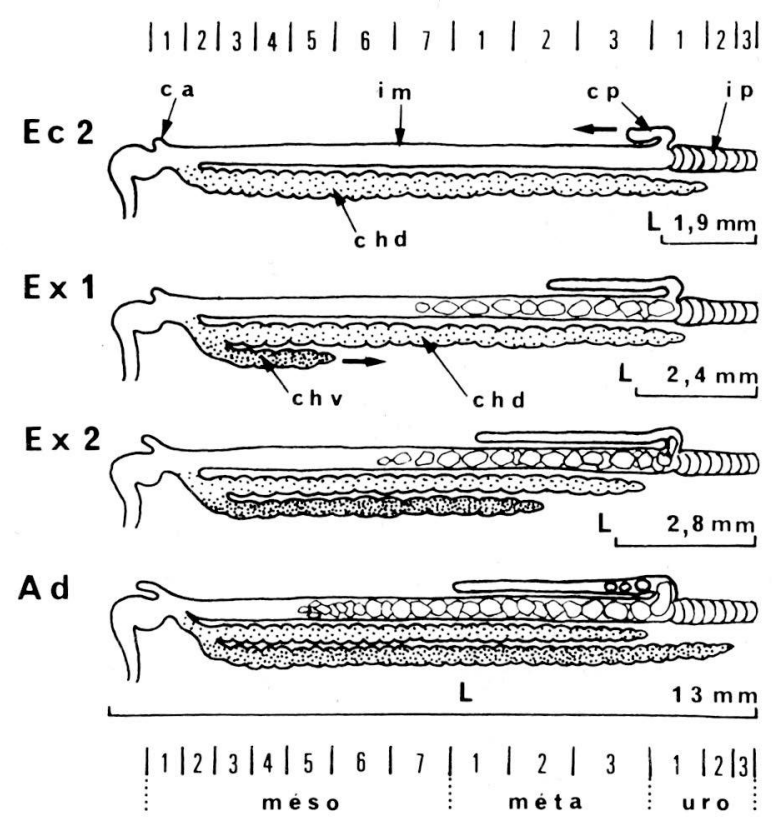

Fig. 4. Représentation schématique de l'état de développement des dérivés de l'intestin moyen lors de 1'éclosion $2(E c 2)$, de l'exuviation 1 ( $E x 1)$, de l'exuviation 2 ( $\left.E x 2_{2}\right)$ et chez l'adulte $(A d)$, par rapport aux segments du mésosome (méso), du métasome (méta) et de l'urosome (uro). Les échelles $(L)$ précisent la longueur des individus aux stades considérés. Chez les jeunes le diamètre des caecums hépatopancréatiques est proportionnellement plus grand que chez les adultes. $c a$, caecum antérieur; $c h d$, caecum hépatopancréatique dorsal; $c h v$, caecum hépatopancréatique ventral; $c p$, caecum postérieur; $i m$, intestin moyen; $i p$, intestin postérieur.

\section{B - Relation des caecums postérieurs avec l'aorte}

La position des caecums postérieurs a intrigué la plupart des auteurs qui les ont observés chez divers Gammaridés (cf.biblio. in Graf, 1969), d'une part du fait de leur position dorsale par rapport au septum péricardique alors que la totalité de l'appareil digestif est localisé dans le compartiment ventral (planche II) et, d'autre part, parce qu'ils paraissent a priori logés dans la lumière de l'aorte postérieure: selon Cussans (1904) et Graf (1969) "les caecums postérieurs sont en contact étroit avec la paroi de l'aorte mais ne sont pas placés dans sa lumière", alors que pour Gibert (1971) "l'aorte postérieure enveloppe complètement ces deux caeca qui sont donc entièrement contenus dans sa lumière interne". Il convenait donc d'étudier la relation des caecums avec l'aorte postérieure non seulement chez des adultes, ce qui a été fait par Klovekorn (1934), mais aussi chez des individus sur le point d'éclore, c'est-à-dire au moment où les caecums se développent (fig. 5). 
L'aorte postérieure est étroitement associée au septum péricardique, elle est en fait localisée dans le septum qui est constitué par l'accolement de deux lames: comme le coeur (planche I, 4 et 5; planche II, 1), l'aorte postérieure s'est vraisemblablement constituée selon le mode de formation du vaisseau dorsal des Annélides, l'espace virtuel compris entre les deux lames septales correspondant à un reliquat de cavité coelomique (?) et la lumière du vaisseau à une cavité blastocoelienne (Weygolt, 1958; Siewing, 1969, p. 231, 322, 326). La lame ventrale du septum est en continuité avec le mésoderme intestinal par l'intermédiaire d'une ou deux connexions mésodermiques (planche I, 4 et 5) qui sont, chez l'adulte, parfois bien visible (planche II, 5) ou le plus souvent noyées dans un tissu adipeux; ces connexions ne sont, bien entendu, pas homologues des mésentères des Annélides ou des Vertébrés. L'aorte postérieure est bien délimitée par une paroi propre de l'extrémité du tube cardiaque jusqu'au niveau de la région antérieure du proctodeum, mais sa localisa-
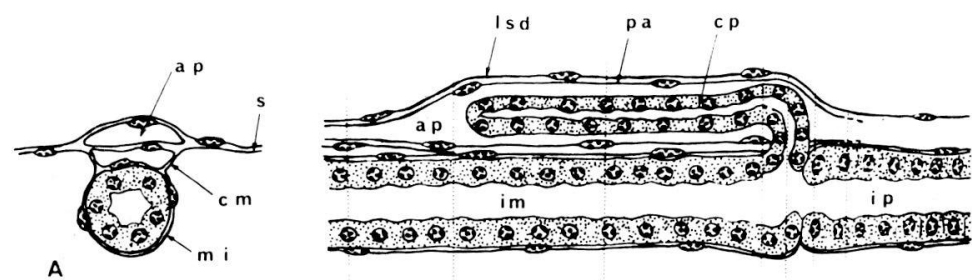

A $\quad$ B

C

DEF G
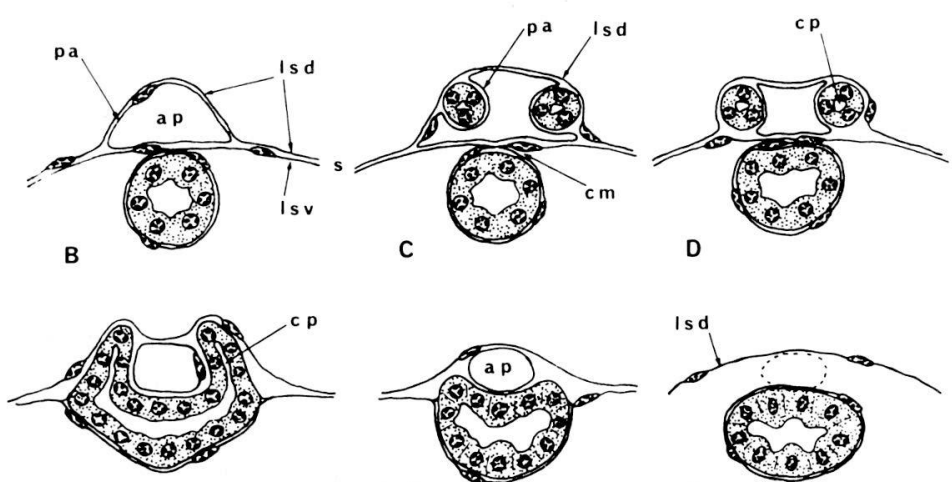

$\mathbf{E}$

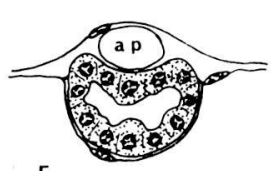

F

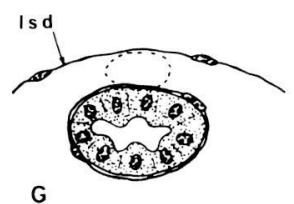

G

Fig. 5. Relation des caecums postérieurs avec l'aorte et le septum péricardique chez un $N$. schellenbergi venant d'éclore. $a p$, aorte postérieure; $\mathrm{cm}$, connexion mésodermique; $c p$, caecum postérieur; im, intestin moyen; $i p$, intestin postérieur; lsd, lame septale dorsale; $l s v$, lame septale ventrale; $m i$, mésoderme intestinal; $p a$, paroi de l'aorte; $s$, septum péricardique. En réalité les lames du septum sont intimement accolées entre elles et avec la paroi de l'aorte. 
tion par rapport au septum parait se modifier au cours de son trajet: en avant de la région distale des caecums l'aorte est localisée dans le plan du septum (fig. $5 \mathrm{~A}$ ), à l'approche et au niveau des caecums elle apparait placée au-dessus du septum (fig. 5, $\mathrm{B}$ à D), alors qu'au niveau de l'intestin postérieur elle est située en dessous (fig. 5,F et $\mathrm{G}$ ). En réalité, comme nous l'avons dit, l'aorte est localisée entre les deux lames septales et au niveau de la zone d'évagination des caecums postérieurs, la lame septale ventrale n'est plus présente alors que la lame septale dorsale persiste sur la plus grande longueur de l'urosome. Lors de leur évagination, les caecums postérieurs dépriment la lame septale dorsale pour s'accoler latéralement ou même dorsalement à l'aorte (fig. 5, E). Ils migrent ensuite vers l'avant entre la paroi de l'aorte et la lame septale dorsale (planche II, 2 à 5), ils ne sont jamais dans la lumière interne de l'aorte, mais présentent cependant un contact très net avec le flot sanguin.

\section{C - Stockage du calcium avant la mue}

\section{Chez l'adulte}

Chez $N$. schellenbergi adulte le stockage de calcium avant la mue est essentiellement localisé dans l'intestin moyen, du mésosomite 5 à l'urosomite 1 , sous forme de rhomboèdres de calcite ou de complexes rhomboédriques (planche III, 3) qui mesurent en moyenne $175 \mu$ de long pour $150 \mu$ d'épaisseur (un complexe de $400 \mu$ x $175 \mu$ a cependant été observé chez un individu de $10,5 \mathrm{~mm}$ ). Dans les caecums postérieurs le stockage calcique n'est jamais très important, il est généralement constitué par trois sphérolithes localisés dans la région proximale de chaque caecum (région antérieure de l'urosomite 1 et parfois région postérieure du métasomite 3), ce qui signifie que les $3 / 4$ antérieurs ne stockent pas de calcium. S'ils ne sont pas nombreux, ces sphérolithes sont cependant relativement gros et peuvent atteindre $175 \mu$ de long et $120 \mu$ d'épaisseur. Il arrive fréquemment d'observer un complexe rhomboédrique arqué, engagé dans la région proximale d'un caecum et dans l'intestin (planche IV, 3). Plus rarement un chapelet de sphérolithes peut être observé dans l'intestin, au milieu des rhomboèdres. Ces deux observations laissent à penser que du calcium sécrété par les caecums pourrait être acheminé dans l'intestin, soit sous forme soluble, soit sous forme de sphérolithes qui évolueraient en forme rhomboédrique par cristallisation secondaire. De cette hypothèse très vraisemblable, déjà envisagée dans le cas de $N$. virei (Graf, 1969), il résulte que l'absence d'un stock calcique important dans les caecums ne signifie pas que ces organes n'ont pas participé à l'élaboration des concrétions intestinales.

\footnotetext{
PLANCHE III.

Concrétions calcaires sous forme sphérolithique (sph) dans les caecums postérieurs $(c p)$ et rhomboédrique $(\mathrm{rh})$ dans l'intestin moyen $(\mathrm{im})$ lors de l'exuviation.

1 - Chez un $N$. virei de $19 \mathrm{~mm}$

2 - Chez un $N$. virei de $9 \mathrm{~mm}$

3 - Chez un $N$. schellenbergi de $13 \mathrm{~mm}$

Ces trois photographies étant reproduites au même grossissement, il est clair que le stockage de calcium est beaucoup moins important chez $N$. schellenbergi et, dans ce cas, quelques sphérolithes seulement sont localisés dans la région proximale des caecums postérieurs.
} 


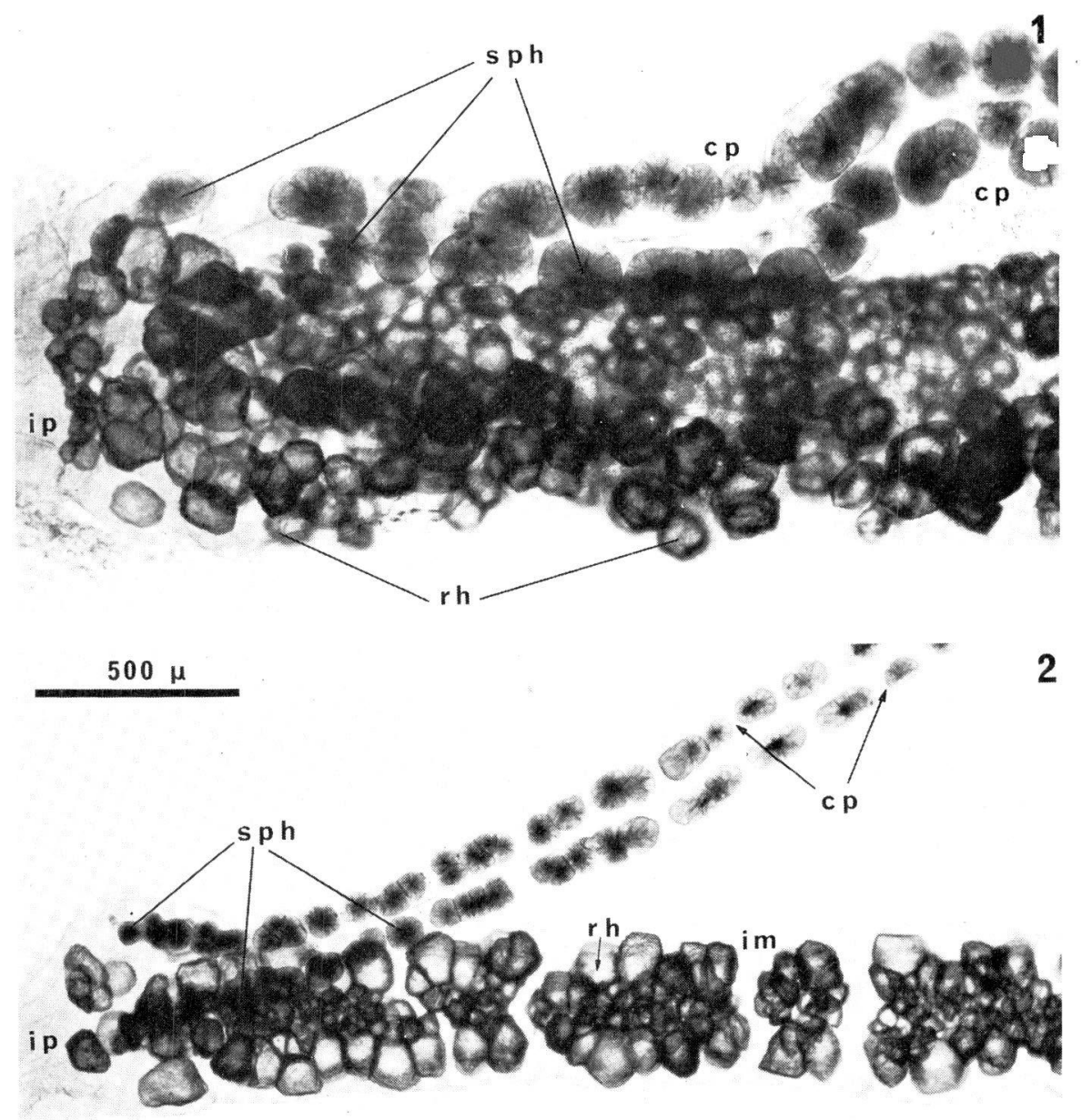

3

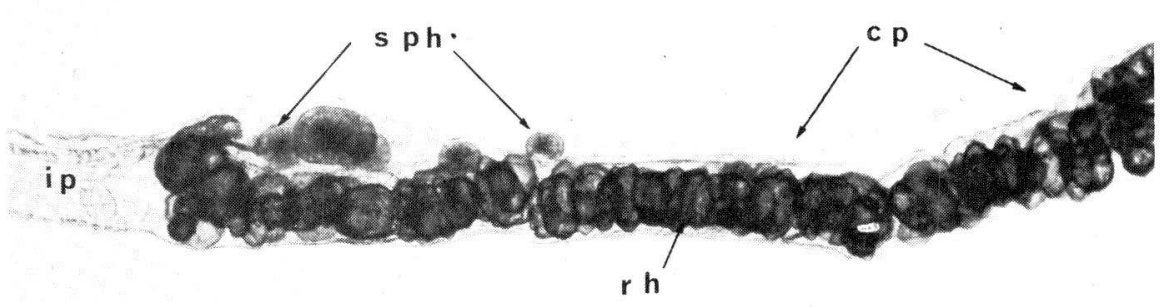


En fait, le stock calcique total de $N$. virei apparait proportionnellement plus important que celui de $N$. schellenbergi, ce qui est lié au fait que le squelette de $N$. virei est plus calcifié que celui de $N$. schellenbergi. Chez $N$. virei, le stock calcique dilate l'intestin et occupe plus des 3/4 de la longueur des caecums (planche III, 1 et 2), alors que chez $N$. schellenbergi l'intestin n'est pas déformé et seulement le $1 / 4$ de la longueur des caecums est susceptible de renfermer des sphérolithes (planche III, 3). Ces constatations sont valables pour des populations diverses vivant dans des eaux dont la teneur en calcium est différente (de 90 à $135 \mathrm{mg} / \mathrm{l}$ ) et sont indépendantes de la présence ou de l'absence de cellules à urates. Il ne semble pas que l'absence de stockage important dans les caecums postérieurs de $N$. schellenbergi puisse être mise en relation avec le fait que le corps de cette espèce est plus grêle et plus aplati que celui de $N$. virei, ce qui pourrait entrainer une compression plus forte de l'aorte postérieure; en effet, le corps de Niphargus foreli Humbert n'est pas beaucoup plus trapu et, chez cette espèce, les caecums postérieurs sont dilatés par de gros sphérolithes (planche II, 6). Chez Niphargus kochianus, les sphérolithes occupent les $3 / 4$ des caecums et sont minuscules $(5 \mu)$ comparativement à ceux des foreli de même taille. Sans entrer dans le détail, il convient de préciser que la forme, le volume, la quantité et la répartition des concrétions calcaires sont caractéristiques de chacune des quatre espèces observées jusqu'à présent, à savoir virei, schellenbergi, foreli et kochianus.

Chez $N$. schellenbergi, la mise en réserve de calcium débute au cours de l'étape D 2, soit une dizaine de jours avant l'exuviation pour des individus de 10 à $13 \mathrm{~mm}$ dont l'intermue totale est de 6 à 9 mois. La dissolution des concrétions commence aussitôt après l'exuviation, elle est généralement totale dans les $48 \mathrm{~h}$ suivantes; l'animal s'alimente dans les $24 \mathrm{~h}$ qui suivent l'exuviation, c'est-à-dire avant que les concrétions soient entièrement solubilisées. Notons que chez certains individus, il apparait dans les caecums de petits sphérolithes de $10 \mu$ maximum, nouvellement formés, alors que la dissolution des autres concrétions est presque terminée, ce qui pourrait être le fait d'une calcémie momentanément trop élevée.

L'élimination de concrétions observée dans certains cas chez $N$. virei (Husson, 1950 b; Graf, 1969) n'a jamais été constatée chez N. schellenbergi.

\section{Au cours du développement}

Contrairement à ce qui a été observé chez Orchestia chez laquelle le stockage de

\section{PLANCHE IV.}

Niphargus schellenbergi au cours des premières mues.

1 - A l'étape D 2 avant la première exuviation, soit quelques jours après la sortie du marsupium. On note la présence de concrétions calcaires rhomboédriques $(r h)$ dans l'intestin moyen et de cellules à urates $(\mathrm{cu})$ au niveau du septum péricardique du mésosome.

2 - En cours de 3ème exuviation, alors que l'intestin moyen renferme des rhomboèdres de calcite $(r h)$, les caecums postérieurs $(c p)$ ne présentent pas de concrétions; $a c$, ancienne cuticule; $i p$, intestin postérieur; $n c$, nouvelle cuticule.

3 - Individu de $5,3 \mathrm{~mm}$ aussitôt après la 6ème exuviation. A ce stade de développement la répartition des concrétions calcaires est semblable à celle de l'adult; $\mathrm{cp}$, caecums postérieurs; $i m$, intestin moyen; $i p$, intestin postérieur; lacp, limite antérieur des caecums postérieurs; $s p h$, sphérolithes. 

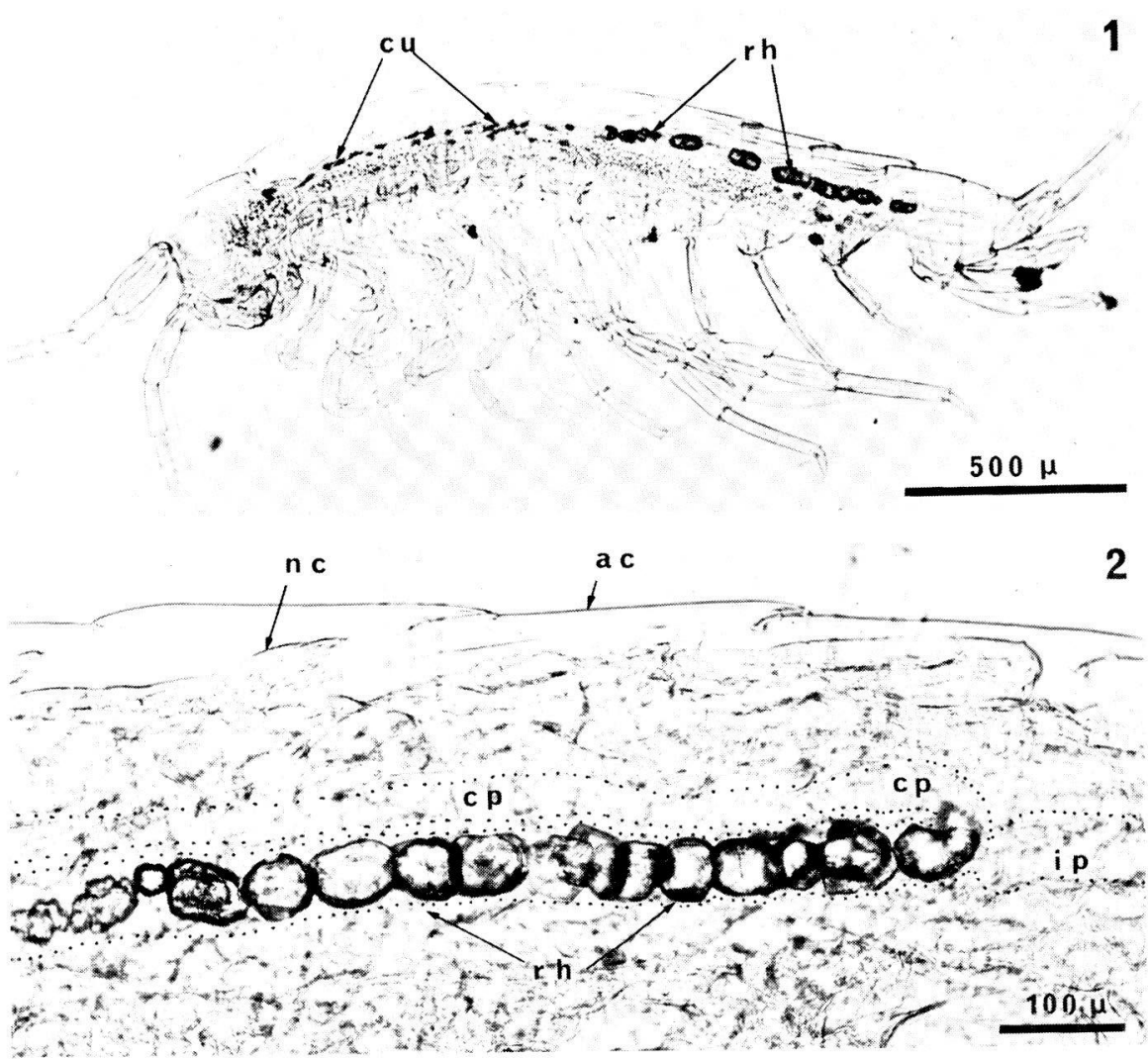

\section{$500 \mu$}

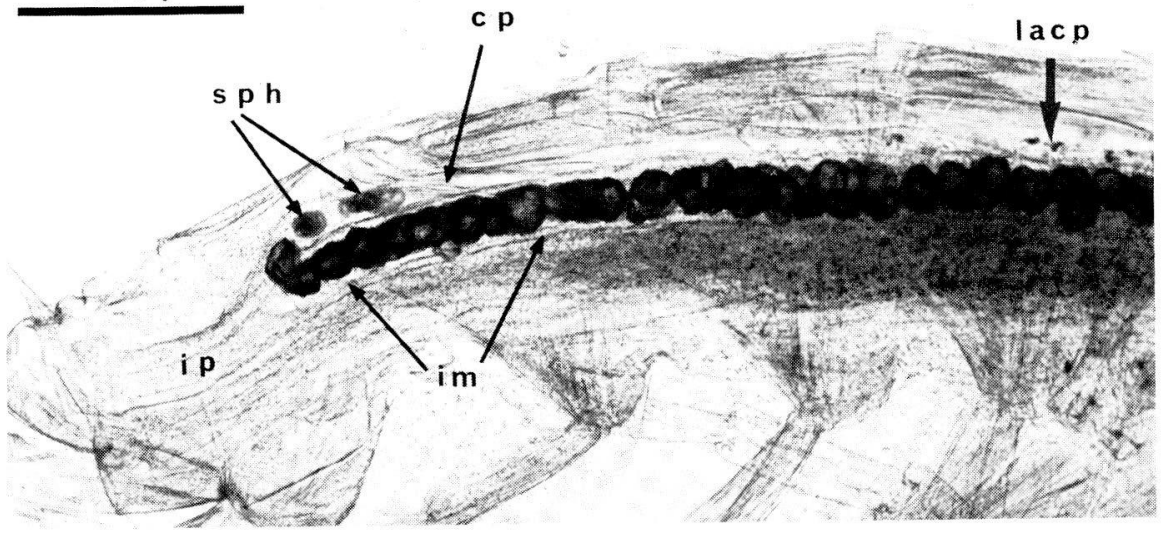


calcium débute au cours de l'intermue embryonnaire (Graf, 1972), il n'y a pas de mise en réserve de calcium lors de l'éclosion 2 de $N$. schellenbergi; à ce moment l'intestin renferme encore du vitellus et les caecums postérieurs n'en sont qu'au début de leur développement. La jeune Orchestia sera libérée dans le milieu "aérien" dans les 4 jours qui suivent cette éclosion, alors que le jeune Niphargus demeurera encore 30 jours à l'abri du marsupium et aura la possibilité de prélever le calcium nécessaire à la calcification de son squelette dans l'eau ambiante. L'absence de stockage de calcium durant la deuxième phase embryonnaire de Niphargus oblige à considérer que la dénomination de cette période comme "intermue embryonnaire" n'est pas aussi justifiée que chez Orchestia, mais mérite cependant d'être conservée pour Niphargus, du fait qu'elle correspond à l'intervalle de temps séparant deux exuviations particulières. Lors de l'exuviation 1 (soit 10 jours après la libération du marsupium), il existe un stock calcique dans l'intestin moyen: une quinzaine de rhomboèdres (70 $\mu \times 50 \mu$ pour les plus développés) répartis du mésosomite 7 au milieu de l'urosomite 1 (planche IV, 1). Ces concrétions sont élaborées dans les $48 \mathrm{~h}$ qui précèdent l'exuviation et sont dissoutes dans les $12 \mathrm{~h}$ qui la suivent. Il n'y a aucune concrétion dans les caecums postérieurs qui ne sont qu'à demi développés.

$\mathrm{Au}$ cours des exuviations suivantes, le nombre des rhomboèdres augmente et le stockage progresse vers la région antérieure de l'intestin, jusque dans le mésosomite 5 (planche IV, 2). Dans les caecums postérieurs, quelques microsphérolithes (d'environ $5 \mu$ ) sont présents dans la région proximale lors de l'exuviation 2 (individus d'environ $3 \mathrm{~mm}$ et 6 mois). Ce n'est que au cours de la deuxième année (individus de $5 \mathrm{~mm}$ ) que le stockage sera semblable à celui des adultes: il faut en effet attendre la sixième ou la septième intermue pour que quelques gros sphérolithes apparaissent dans la région proximale des caecums (planche IV, 3).

Ainsi, comparativement à Orchestia où la mise en réserve de calcium dans les caecums postérieurs débute dès l'intermue embryonnaire et est d'emblée semblable à celle de l'adulte, chez $N$. schellenbergi ce processus ne débute qu'en vue de l'exuviation 1 et s'établit progressivement au cours de la première année; cette différence est vraisemblablement due au fait que chez le Talitridé les caecums se développent totalement au cours de l'intermue embryonnaire, alors que leur développement chez $N$. schellenbergi ne commence qu'au moment de l'éclosion et qu'il est relativement lent. D'après quelques observations fragmentaires effectuées chez $N$. virei, les caecums postérieurs se développent aussi lentement que chez schellenbergi, mais nous ignorons à quel moment ils commencent à stocker des sphérolithes calcaires.

\section{EVOLUTION DES CELLULES à URATES.}

La présence de cellules à urates chez un Amphipode semble avoir été reconnue en premier chez $N$. schellenbergi (Graf, 1971). Ce sont des cellules jaunâtres qui sont le plus souvent localisées au niveau de la tête et du mésosome (planche V). Observées en grand nombre chez des $N$. schellenbergi d'une même 
population, ces cellules ont été recherchées dans d'autres populations de la même espèce et d'espẹ̀ces différentes: il résulte de cette étude que des populations de la même espèce en sont dépourvues, alors que des espèces différentes en possèdent. C'est ainsi que $N$. virei en présente généralement au niveau de la tête et que dans une population de $N$. foreli (thienemanni?) (récoltée par J.P. HENRY dans le sous-écoulement de la rivière la Loue, Jura) ces cellules à urates sont très nombreuses et réparties tout le łong du corps (planche II, 6).

En fait ces cellules avaient déjà été observées. C'est ainsi que dans les généralités sur le genre Niphargus, Schellenberg (1942, p. 51) note l'absence d'yeux et de pigments du corps, si ce n'est parfois une coloration rose pâle ou jaune et la présence d'un endroit jaune ou orange à l'emplacement des yeux ("und einem gelben oder orange Flecken in der Augengegend"). Ces cellules jaune citron ou jaune soufre ont d'ailleurs été interprétées comme des yeux par C.Sp.Bate (in Vejdovsky, 1896) ou comme des reliquats de pigments oculaires (Vejdovsky, 1896, 1905). Cet auteur note que le pigment est jaune mais peut apparaitre blanc, brun ou noir selon le mode d'éclairage, et décrit des cas de réductions oculaires où il retrouve ces cellules pigmentaires au milieu des cônes cristallins dispersés et plus ou moins nombreux au niveau de la tache oculaire. Il s'interroge cependant quant à la fonction de ces cellules pigmentaires dont la présence, la quantité ou l'aspect ne sont pas identiques des deux côtés.

Chez les Isopodes, les organes de Zenker d'Asellus et les organes jaunes de Caecosphaeroma sont constitués de cellules homologues des cellules à urates de Niphargus (cf.biblio.in Graf, 1971).

\section{A - Localisation}

Les cellules à urates sont accolées à la face inférieure du septum péricardique (planche III, 1 et 2) et font, de ce fait, saillie dans le compartiment médian, entre le tube digestif et les caecums hépatopancréatiques. Elles peuvent aussi être disposées au niveau des membranes intersegmentaires des premiers mésosomites et des vaisseaux péricardiques (planche $\mathrm{V}, 1$ et 5) qu'elles longent parfois jusque dans les basipodites des péréiopodes. Chez $N$. foreli, elles peuvent s'étendre jusqu'au niveau de l'urosome, de part et d'autre des caecums postérieurs et plus précisément de l'aorte (planche II, 6). Ces cellules diffèrent des néphrocytes péricardiaux décrits chez divers Amphipodes (Bruntz, 1903) mais non retrouvés chez Niphargus (Husson, 1951) et qui sont des athrocytes localisés autour du coeur.

Sur l'animal vivant ces cellules sont opaques, leur teinte pouvant varier d'un blanc légèrement jaunâtre à un jaune vif. Chez l'animal fixé, elles apparaissent sombres par transparence (planche $\mathrm{V}, 7$ ) et d'un blanc brillant en observation en fond noir (planche V, 2 à 6). Les concrétions qu'elles renferment étant solubilisées par les fixateurs aqueux (y compris les alcools $70^{\circ}$ ou $90^{\circ}$ ), il convient, pour les conserver, d'utiliser un fixateur anhydre, tel que l'alcool-chloroforme (2-1) et d'entreposer les échantillons dans un congélateur. Pour une étude histologique, il est nécessaire, d'une part d'employer le même fixateur ou le liquide de Carnoy et, d'autre part, d'insolubiliser les sphérolithes avant d'utiliser des colorants aqueux, soit par le complexe d'argento-méthénamine, soit par la méthode de von Kossa. 


\section{B - Constitution}

Les cellules à urates sont très irrégulières (planche $\mathrm{V}, 6$ ), elles présentent des prolongements ramifiés parfois ténus qui s'insinuent entre les cellules adipeuses (planche $\mathrm{V}, 7$ ). Elles mesurent de 50 à $150 \mu$ (noyau de $10 \mu$ environ) et sont chargées de microsphérolithes de 0,5 à $3 \mu$ dont la structure est à la fois radiaire et concentrique. Ces sphérolithes sont plus réfringents que le baume de Canada et polarisent dans les teintes de premier ordre en présentant une croix noire très nette. L'étude microchimique de ces concrétions (Graf, 1971) a permis de préciser qu'elles sont constituées d'urates; l'analyse ionique par spectrographie des rayons X, effectuée à la microsonde Cameca grâce à l'obligeance de Pr. R. Martoja, a révélé la présence de phosphore, potassium, calcium, soufre, chlore, sodium et magnésium, ions généralement associés aux urates du corps gras de certains Insectes (Ballan-Dufrançais et Martoja, 1971; Martoja, 1971). Quant aux pigments jaunes, qui colorent parfois intensément les cellules à urates, il est vraisemblable qu'ils appartiennent au groupe des ptérines qui ont été mises en évidence dans les organes jaunes de Caecosphaeroma (Descimon et Marvillet, 1966).

\section{C - Evolution}

Les divers auteurs (cf. biblio. in Husson et Henry, 1963) qui ont étudié les organes de Zenker ou les organes jaunes des Isopodes, leur attribuent une fonction d'excrétion azotée, en considérant ces "organes" comme des reins d'accumulation de déchets puriques provenant du catabolisme protéique. Il convient en fait de savoir si ces cellules sont des sites d'accumulation définitive de déchets puriques ou des lieux de stockage temporaire d'urates, phosphates, pigments et ions divers. Pour tenter de répondre à cette question, nous avons cherché à préciser, d'une part à quel moment apparaissent ces cellules et, d'autre part, si elles se caractérisent ou non par une stabilité de leur stucture ou de leur composition.

Chez $N$. schellenbergi, les cellules à urates commencent à se charger de sphérules au cours du développement embryonnaire, 15 jours avant l'éclosion, c'est-à-dire

\section{PLANCHE V.}

1 - Répartition des cellules à urates chez un $N$. schellenbergi mâle de $14 \mathrm{~mm}$.

2 - Embryon de $N$. schellenbergi une dizaine de jours avant l'éclosion 2 ; les cellules à urates (flèches) sont bien visibles en éclairage en fond noir (échelle: $100 \mu$ ).

3 - Détail des cellules à urates de la photographie précédente. Dix jours avant l'éclosion, ces cellules renferment de nombreuses sphérules d'urates (échelle: $100 \mu$ ).

$4-N$. schellenbergi en intermue 1 dans le marsupium, soit une vingtaine de jours après l' éclosion. Les cellules à urates sont plus nombreuses qu'au stade précédent (échelle: $100 \mu$ ). 5 - Répartition des cellules à urates dans le péréion d'un mâle de $14 \mathrm{~mm}$ au niveau du septum péricardique $(s)$, des vaisseaux péricardiques (flèches), des membranes intersegmentaires (astéris-
ques) (échelle: $500 \mu$ ).

6 - Cellules à urates dans le septum péricardique vu à plat; les flèches indiquent l'axe du coeur (échelle: $100 \mu$ ).

7 - Cellule à urates dans laquelle on distingue l'emplacement du noyau $(n)$ et la présence de sphérules d'urates dans les prolongements (flèches) qui s'insinuent entre les cellules adipeuses (échelle: $50 \mu$ ). 

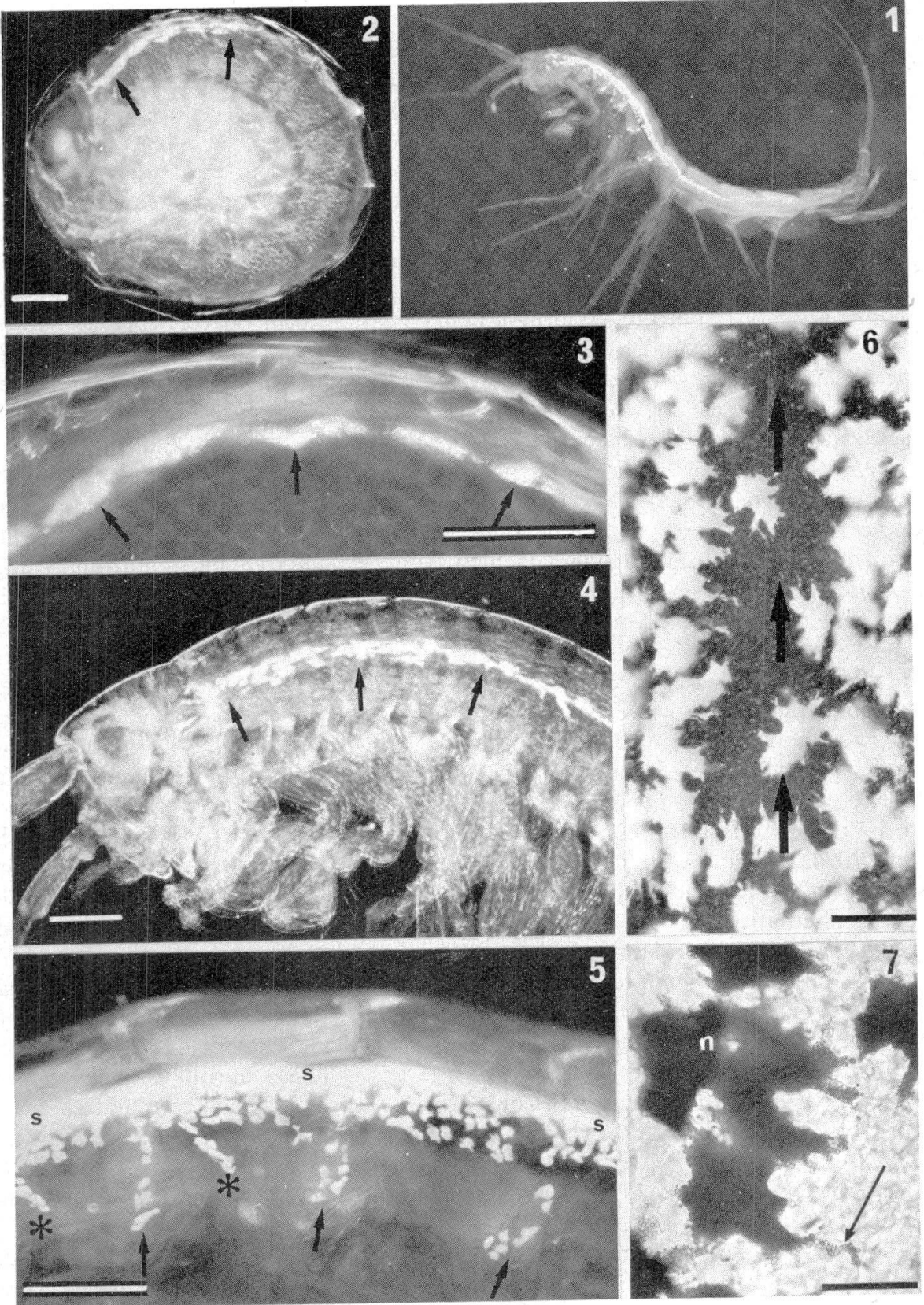
quelques jours avant que ne soit établie la circulation sanguine. Ces cellules, au nombre d'une trentaine environ, se localisent tout d'abord au niveau du septum péricardique des 4 premiers segments du mésosome (planche $\mathrm{V}, 2$ ); elles sont généralement étirées et mesurent en moyenne $60 \mu$, taille déjà importante. Les sphérolithes apparaissent progressivement dans le cytoplasme de ces cellules (planche V,3) et il est net que le volume définitif de la cellule chargée de sphérules n'est pas conditionné par la quantité accumulée mais qu'il correspond au volume initial. Il faut donc considérer que le volume important de ces cellules est une prédisposition au stockage et non une conséquence de l'accumulation. Avec la croissance de l'animal, le nombre et le volume des cellules peuvent augmenter (planche V, 4 et 6), mais cette augmentation se fait proportionnellement au développement du corps entier et les variations de quantité d'urates que l'on peut oberver chez les adultes s'observent également à tous les stades du développement. L'accumulation d'urates chez $N$. schellenbergi n'est donc pas évidente, elle serait plus apparente chez Asellus, mais Dresel et Moyle (1950) indiquent que, pour des individus de poids différents, la proportion d'acide urique est assez constante.

En fait, au sein d'une même population de $N$. schellenbergi, la quantité de cellules à urates est extrêmement variable suivant les individus et la question qui se pose est de savoir s'il existe de telles variations au cours de la vie d'un même individu. L'observation régulière pendant 10 mois d'une trentaine d'individus élevés en présence ou en absence d'argile, dans de l'eau provenant de la station de récolte, soit naturelle, soit plus ou moins diluée à l'eau distillée ou concentrée à l'eau de mer, abondamment nourris ou, au contraire, en état de jeûne, montre que la quantité totale d'urates varie très lentement; elle diminue chez certains, augmente chez d'autres, sans qu'il soit possible d'établir une corrélation entre le mode d'élevage et ces variations, qui se manifestent essentiellement par des rétractions ou des extensions des cellules et des modifications dans la teinte plus ou moins jaune des pigments. L'observation plus précise d'un petit groupe de cellules d'un individu élevé dans des conditions normales est plus instructive. On constate en effet, comme le montre la figure 6, que non seulement les contours cellulaires se déforment sans cesse, mais encore que ces cellules changent de position. En général, chaque cellule passe alternativement par des phases d'extension, des prolongements cytoplasmiques chargés de sphérules s'insinuant alors entre les cellules adipeuses (planche V, 7) et par des phases de rétraction. Dans les conditions normales d'élevage, l'animal présente généralement autant de cellules rétractées que de cellules en extension et, de ce fait, la quantité totale d'urates semble ne pas varier, alors que, dans des conditions expérimentales, c'est la majorité des cellules qui sont dans un état ou l'autre, ce qui entraine des variations perceptibles de la quantité d'urates. Ainsi, chez $N$. schellenbergi, les variations de volume de chaque cellule à urates démontrent qu'une reprise des urates stockés est possible et les modifications importantes et relativement rapides de la surface cellulaire sont un des signes d'un métabolisme cellulaire actif.

Quant à la constitution de la charge des cellules à urates, la seule observation de variations dans la teinte des pigments est déjà une preuve d'une modification de leur composition ou de leur teneur. Il convient de signaler à ce sujet que, dans une 
population de $N$. foreli récoltée en mars, les cellules à urates étaient jaune franc chez les mâles et très peu pigmentées chez les femelles. De plus, après une dessiccation prolongée, les cellules à urates apparaissent claires, brunes ou noires ce qui correspond vraisemblablement à des états successifs de l'évolution de leur composition. Afin de rechercher d'éventuelles variations dans la teneur ionique, l'analyse par spectrographie des rayons $\mathrm{X}$ a été conduite sur des cellules claires, brunes et noires de trois $N$. schellenbergi, un mâle, une femelle et un mâle élevé durant un mois dans une solution de $\mathrm{NaCl}$ à 6/1000. Les résultats de cette analyse (fig. 7) montrent qu'il existe des variations dans la teneur ionique entre les cellules d'un même individu et celles d'individus différents. Si ces données quantitatives, du fait qu'elles ne portent que sur 3 individus, ne sont pas suffisantes pour autoriser des hypothèses quant au rôle de ces cellules, elles permettent cependant d'affirmer que le stock des cellules à urates est susceptible de variations quantitatives et qualitatives: c'est ainsi que phosphore et calcium présentent des variations inverses et que chez l'individu élevé dans une solution de $\mathrm{NaCl}$, la perte de phosphore et de potassium est évidente.
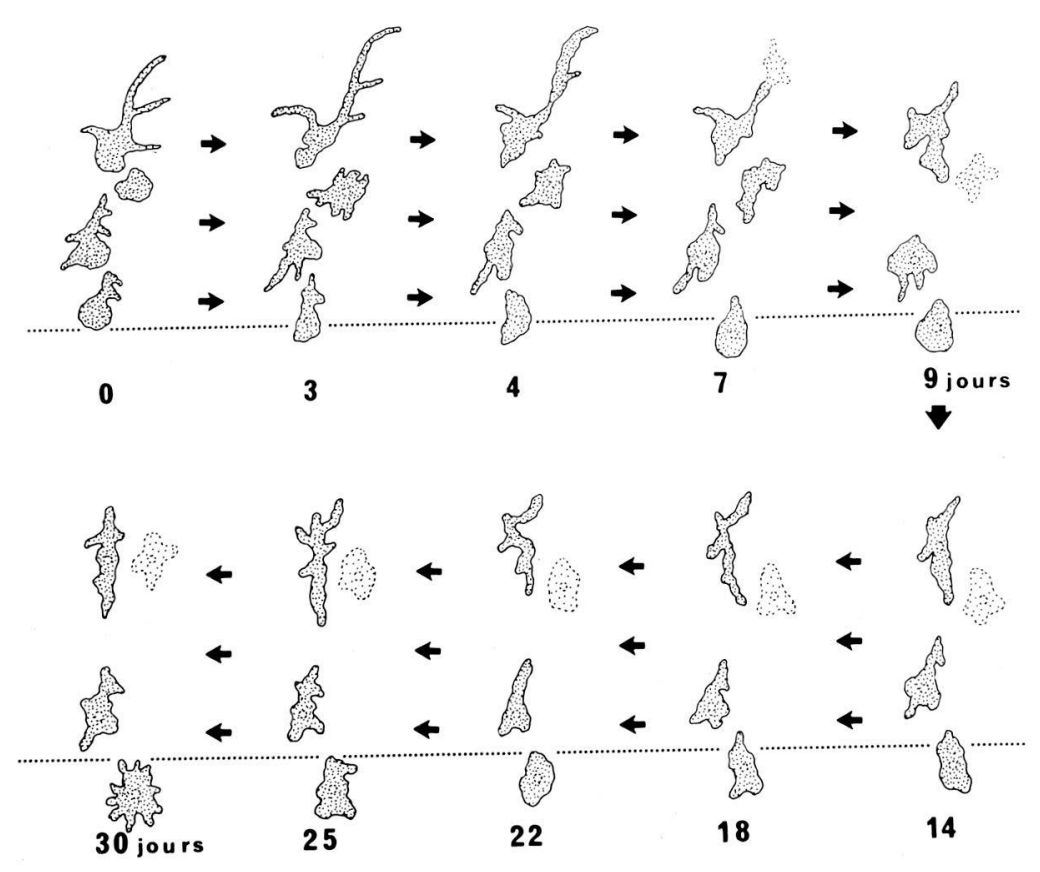

Fig. 6. Modifications de la forme de quatre cellules à urates observées durant 30 jours au niveau du mésosomite 5 d'un $N$. schellenbergi. La droite en pointillé correspond à la limite supérieure de la plaque coxale. Les contours cellulaires représentés en pointillé indiquent qu'ils sont localisés dans un plan sous-jacent au plan d'origine. 
Ainsi, chez $N$. schellenbergi, les cellules à urates peuvent être considérées non pas comme des reins d'accumulation des déchets du catabolisme protéique, mais comme des sites de stockage d'urates, de pigments et de divers ions qui pourraient être remis en circulation. On sait en effet, d'une part que chez les Insectes l'acide urique peut constituer une réserve azotée réutilisable et participer à la synthèse d'acides nucléiques et d'acides aminés (Mitlin et Wiygul, 1973), et, d'autre part, que des pigments jaunes possédant le noyau ptéridine peuvent avoir une grande importance comme coenzymes d'oxydation (Viscontini et Schmidt, 1965).
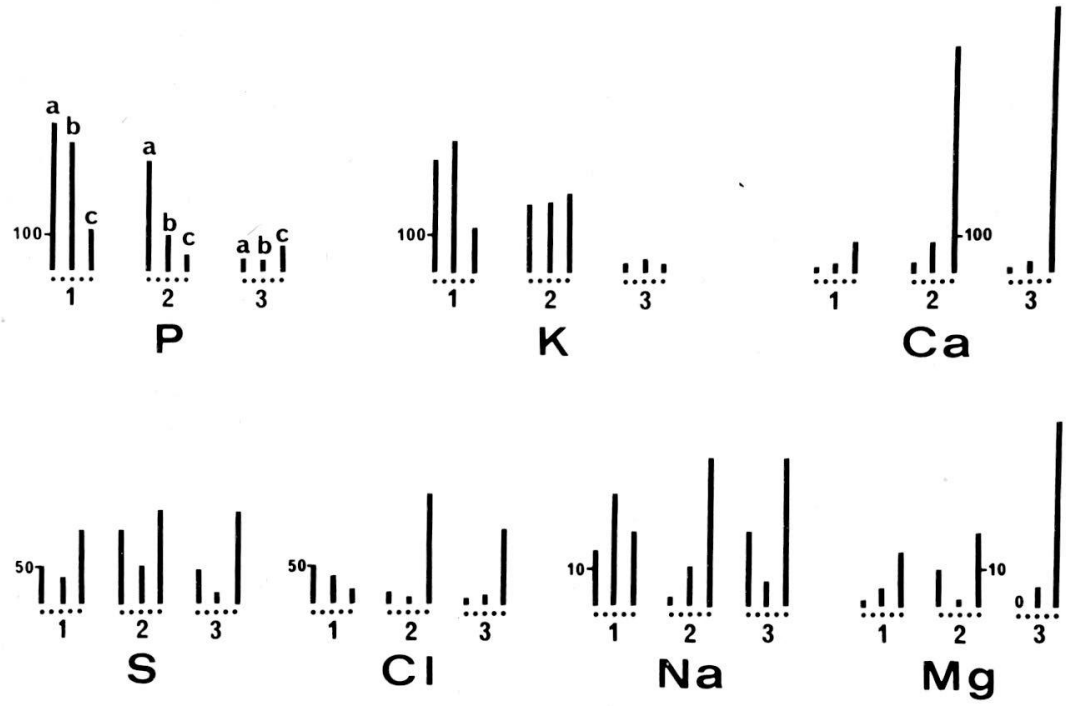

Fig. 7. Teneur en ions $\mathrm{P}, \mathrm{K}, \mathrm{Ca}, \mathrm{S}, \mathrm{Cl}, \mathrm{Na}$ et $\mathrm{Mg}$ des cellules à urates de trois $N$. schellenbergi: un mâle (1), une femelle (2) et un mâle élevé durant un mois dans une solution $\mathrm{NaCl}$ à 6/1.000. (3). Pour chaque individu l'analyse a été faite sur des cellules claires $(a)$, brunes (b) et noires $(c)$. Les nombres portés en ordonnées correspondent à l'intensité exprimée en nombre de chocs/seconde. Les teneurs diverses ne sont comparables quantitativement que pour un même ion.

\section{RESUME}

Le développement intra-marsupial de Niphargus schellenbergi peut être subdivisé en trois phases: la phase du chorion, l'intermue embryonnaire et l'intermue juvénile. Les deux premières phases sont séparées par la disparition du chorion, les deux dernières par une double exuviation. La chronologie du développement embryonnaire et du début du développement post-embryonnaire est établie. Lors de l'éclosion le jeune $N$. schellenbergi n'est pourvu que d'une seule paire de caecums hépato- 
pancréatiques; c'est durant les premières intermues post-embryonnaires que seront édifiés les caecums hépatopancréatiques ventraux, les caecums postérieurs et le caecum antérieur. La relation des caecums postérieurs avec l'aorte est décrite.

L'étude du stockage de calcium avant la mue montre que la forme, le volume, la quantité et la répartition des concrétions calcaires dans les caecums postérieurs et l'intestin moyen sont caractéristiques de l'espèce. Ce processus de mise en réserve de calcium survient dès la première exuviation post-embryonnaire dans l'intestin moyen, mais ce n'est que lors de la sixième exuviation que le stockage dans les caecums postérieurs sera semblable à celui des adultes.

Les cellules à urates, localisées à la face inférieure du septum péricardique, commencent à se charger de sphérules d'urates 15 jours avant l'éclosion. Elles présentent des variations de forme, de volume, de position et de constitution et, de ce fait, peuvent être considérées comme des sites de stockage d'urates, de pigments et de divers ions $(\mathrm{P}, \mathrm{K}, \mathrm{Ca}, \mathrm{S}, \mathrm{Cl}, \mathrm{Na}, \mathrm{Mg}$ ) qui pourraient être remis en circulation.

\section{BIBLIOGRAPHIE}

BALLAN-DUFRANCAIS, Ch. 1971: Analyse chimique d'inclusions minérales par spectrographie des rayons $X$ et par cytochimie. Application à quelques organes d'Insectes Orthoptères. J. Micr., 11, 219-248.

BREGAZZI, P. K. 1973: Embryological development in Tryphosella kergueleni (Miers) and Cheirimedon femoratus (Pfeffer). Br. Antarct. Surv. Bull., 32, 63-74.

CUSSANS, M. 1904: Gammarus. L. M. B. C. Memoirs no12. Proc. Liverpool Biol. Soc., 18, 327-373.

DESCIMON, H. \& MARVILLET, Cl. 1966: Mise en évidence de substances fluorescentes dans les organes jaunes de Caecosphaeroma burgundum Dollfus, Crustacé Isopode des eaux souterraines. Int. J. Speleol., 2, 449-455.

DRESEL, E. \& MOYLE, V. 1950: Nitrogenous excretion of amphipods and isopods. J. Exp. Biol., 27, 210-225.

GIBERT, J. 1971: Appareil circulatoire et circulation sanguine de Niphargus virei (Crustacé Amphipode hypogé). Bull. Soc. Zool., Fr., 96, 33-43.

GINET, R. 1955: Etudes sur la biologie d'Amphipodes troglobies du genre Niphargus. I. Le creusement de terriers; relations avec le limon argileux. Bull. Soc. Zool. Fr., 80, 332-349.

GINET, R. 1960: Ecologie, éthologie et biologie de Niphargus (Amphipodes Gammaridés hypogés). Ann. Spéléol., Fr., 15, 127-376.

GOUNOT, A. M.. 1960: Recherches sur le limon argileux souterrain et sur son rôle nutritif pour les Niphargus. Ann. Spéléol., Fr., 15, 501-526.

GRAF, F. 1962: Mise en réserve de calcaire avant la mue chez les Crustacés Amphipodes. $C$. $R$. Acad. Sci., Fr., 255, 2191-2193.

GRAF, F. 1968: Le cycle d'intermue du Crustacé hypogé Niphargus virei Chevreux (Amphipoda, Gammaridae). C. R. Acad. Sci., D, Fr., 266, 823-825.

GRAF, F. 1969: Le stockage de calcium avant la mue chez les Crustacés Amphipodes Orchestia (Talitridé) et Niphargus (Gammaridé hypogé). Thèse Doct. Sc. nat., Dijon, Impr. F. Berthier, Arch. orig. Centre Document. CNRS, no 2690, 1-216.

GRAF, F. 1971: Présence de cellules à urates chez l'Amphipode hypogé Niphargus. C. R. Acad. Sci., D, F., 272, 2239-2242.

GRAF, F. 1972: Stockage de calcium et formation des soies chez l'embryon d'Orchestia (Crustacé, Amphipode, Talitridé). Notion d'intermue embryonnaire. C. R. Acad. Sci., Fr., $D, 275,1669-1672$. 
HUSSON, R. 1949: Observations biologiques sur l'Amphipode Niphargus virei Chev. (note préliminaire). Bull. Soc. Zool., Fr., 74, 126-132.

HUSSON, R. 1950a: Observations biologiques sur l'Amphipode Niphargus virei Chev. (deuxième note). Bull. Soc. Zool., Fr., 75, 211-217.

HUSSON, R. 1950b: Elimination de calcaire avant la mue chez Niphargus virei Chev. Ann. Sci. nat., Zool., Fr., 12, 309-313.

HUSSON, R. 1951: Etude du phénomène d'athrocytose chez un Amphipode cavernicole, $\mathrm{Ni}$ phargus virei Chevreux. Ann. Sci. nat., Zool., Fr., 13, 417-426.

HUSSON, R. 1953: Considérations sur la biologie des Crustacés cavernicoles aquatiques. $C$. $R$. Congr. Intern. Spéléo., Paris, 3, 65-71.

HUSSON, R. 1959: Les Crustacés Péracarides des eaux souterraines. Considérations sur la biologie de ces cavernicoles. Bull. Soc. Zool., Fr., 84, 219-231.

HUSSON, R \& HENRY, J. P. 1963: Etude chez trois espèces du genre Asellus des concrétions des organes de Zenker. Bull. Soc. Zool., Fr., 88, 274-277.

KLOVEKORN, J. 1934: Das Organsystem der Blutbewegung bei Gammarus pulex. Z. Wiss. Zool., 146, 153-192.

MARTOJA, R. 1971: Données préliminaires sur les accumulations de sels minéraux et de déchets du catabolisme dans quelques organes d'Arthropodes. C. R. Acad. Sci., D, Fr., 273, 368-371.

MITLIN, N. \& WIYGUL, G. 1973: Uric acid in nucleic and amino acid synthesis in the boll weevil, Anthonomus grandis. J. Insect Physiol., 19, 1569-1574.

NATH, C. N. 1972: On the storage of calcium in Spelaeomysis, a subterranean mysid. Crustaceana, suppl. 3, 351-353.

SCHELLENBERG, A. 1942: Krebstiere oder Crustacea. IV.: Flohkrebse oder Amphipoda. Tierwelt Deutschlands, 40, Fischer, Iéna, 1-252.

SIEWING, R. 1969: Lehrbuch der vergleichenden Entwicklungsgeschichte der Tiere. Paul Parey, Hamburg, 1-531.

STRASKRABA, M. 1972: L'état actuel de nos connaissances sur le genre Niphargus en Tchècoslovaquie et dans les pays voisins. Actes du 10 Coll. Inter. sur le genre Niphargus. Museo civico di storia naturale di Verona. Memorie fuori serie no 5, 35-46.

TURQUIN, M. J. 1967: L'organe dorsal de Niphargus virei. Spelunca, Mém., 5, 305-312.

TURQUIN, M. J. 1969: Le développement du système nerveux de Niphargus virei. Bull. Soc. Zool., Fr., 94, 649-656.

VEJDOVSKY, F. 1896: Ueber einige Süsswasser-Amphipoden. I. Ueber Crangonyx Sp. Bate. Sitz. Kön. Böhm. Ges. Wiss. Prag., 10, 1-32.

VEJDOVSKY, F. 1905: Ueber einige Süsswasser-Amphipoden. III. Die Augenreduktion bei einem Gammariden aus Irland und über Niphargus Caspary Pratz aus den Brunnen von München. Sitz. Kön. Böhm.Ges. Wiss. Prag., 18, 1-40.

VISCONTINI, M. \& SCHMIDT, G. H. 1965: Ueber die physiologische Bedeutung der Pterine. Z. für Naturforsch., 20, 327-331.

WEYGOLT, P. 1958: Die Embryonalentwicklung des Amphipoden Gammarus pulex (L.) Zool. Jb. Anat., 77, 51-110. 